\title{
Das Optimum herausholen
}

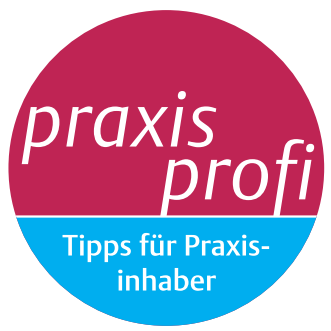

Krisenmanagement Von den Auswirkungen der Corona-Pandemie waren und sind auch die Therapiepraxen ernsthaft betroffen. Wie geht man mit einer solchen unerwarteten Krise um? Und was kann man tun, um daran nicht zu verzweifeln?

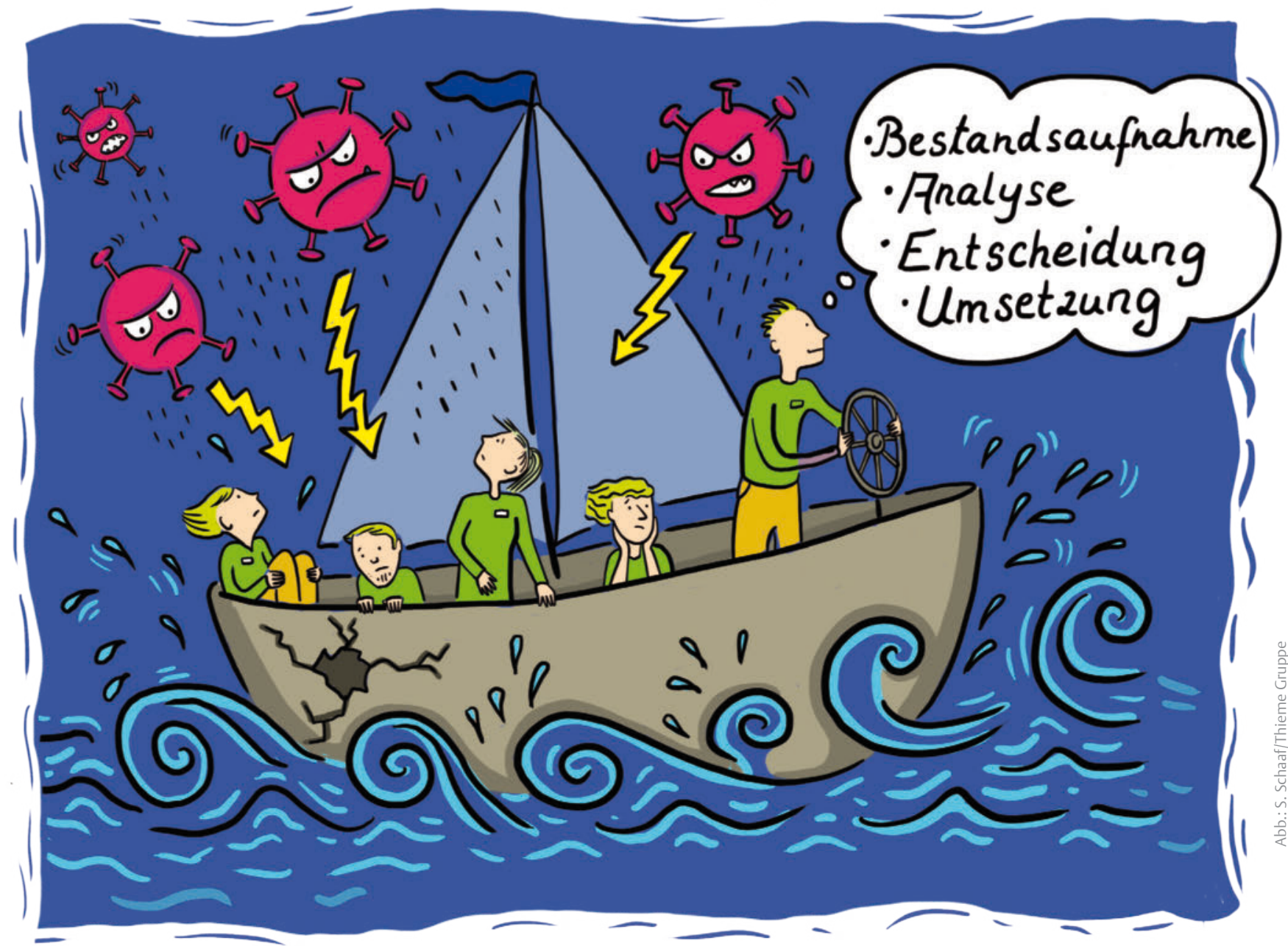

Steuern Sie Ihr Schiff samt den Mitarbeitern als „Captain Therapist“ sicher durch Krisen.

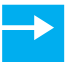

Was für eine Zeit! Vermutlich wird die Situation jetzt, wo Sie diesen Artikel lesen, schon wieder eine völlig andere sein als in dem Augenblick, in dem ich diesen Text schreibe. Wie wird es sein in sechs bis acht Wochen? Haben wir eine zweite, dritte Welle an COVID19-Infektionen? Wird es einen zweiten „Lockdown“ geben, in dem alles geschlossen wird und wir erneut auf Aussagen der Ämter warten, ob therapeutische Praxen geöffnet haben oder geschlossen werden? Wird sich alles in Luft aufgelöst haben und wir blicken erschöpft zurück auf die sicherlich anstrengendste Zeit als Praxisinhaber? Diese Fragen kann gerade niemand beantworten ... Und genauso geht es uns nun schon seit ein paar Monaten.
Seit dem Auftreten der Corona-Krise und den damit einhergehenden Maßnahmen, Vorschriften und Empfehlungen steht unser Leben als Therapeuten und erst recht als Chefs vollkommen auf dem Kopf. Wir wissen nicht, welche Änderungen es in 14 Tagen geben wird. Schulen offen, Schulen geschlossen? Gruppen ja, Gruppen nein? Pflegeheime? Wenn ja, wie? 
Hygienebedarf? Ich lehne mich wohl nicht sehr weit aus dem Fenster, wenn ich behaupte, dass es unfassbar anstrengend, zermürbend und auch beängstigend ist, so wenig Planungssicherheit zu haben wie im Augenblick. Auf was können wir also zurückgreifen? Welche Führungskompetenzen sind genau jetzt wichtig und erforderlich?

\section{5 \\ Verantwortliches Han- deln bedeutet auch, unangenehme Entschei- dungen zu treffen.}

Besinnen wir uns auf unsere Kernkompetenzen $\rightarrow$ Wir sind in der großartigen Situation, dass wir uns auf das besinnen können, was wir Therapeuten gelernt haben: Unterstützung in Krisen und kreative Lösungen finden. So dürfen wir uns gerade jetzt einmal sehr liebevoll selbst als Klienten annehmen und uns in den Fokus nehmen. Tun wir das nicht, kann es schnell geschehen, dass wir hin- und hergeworfen werden zwischen wissenschaftlich widersprüchlichen Aussagen und dem politischen Newsticker, ähnlich einer Nussschale auf hoher See. Das ist ja ohnehin eins meiner Lieblingsbilder, wenn es um das Thema Führung geht, also bleiben wir mal dabei.

Unser Schiff - also die Praxis, das Unternehmen oder auch die Abteilung - wird gerade, ohne dass wir es durch eine Wetter-App, Windfinder oder Ähnliches hätten ahnen können, von einem heftigen Sturm aus heiterem Himmel getroffen. Stellen Sie sich jetzt bitte einmal vor, Sie wären nicht Kapitän, sondern Passagier auf diesem Schiff, zusammen mit den anderen Passagieren, also mit Ihren Mitarbeitern und Ihren Patienten und Klienten. Was würden Sie sich als Passagier auf einem so sturm- und wellengebeutelten Schiff wünschen? Sie wissen, dass der Kapitän keine Möglichkeiten hatte, das Vorgehen langfristig zu planen. Sie wissen, dass dies ein Unwetter ist, das es so bisher noch nicht gab und für das niemand einen Plan B aus der Hosentasche hervorziehen kann. Und Sie wissen, dass auch der Kapitän keine Ahnung haben kann, wie lange das Ganze noch dauern, welche Auswirkungen es haben wird und wie Sie alle aus dieser Ge- schichte herauskommen werden. Ihr Kapitän hat, genau wie alle anderen Kapitäne, mit dieser Situation keinerlei Erfahrung.

Ich glaube zu wissen, was Sie sich dann NICHT wünschen: Sie möchten auf so einem Schiff sicherlich nicht, dass sich der Kapitän zu Ihnen setzt und mit Ihnen gemeinsam ängstlich aus dem Bullauge lugt. Und mit ziemlicher Sicherheit möchten Sie auch keinen Kapitän, der Ihre Sorgen wegwischt, ignoriert oder aber Horrorszenarien à la „Titanic“ ausmalt.

\section{Schritt für Schritt zur Lösung $\rightarrow$ Nun malen} wir das Bild eines vertrauenerweckenden Seebären namens „Captain Therapist“, der diese Krise nicht nur bravourös meistert, sondern auch noch das Optimale an Erfahrungen aus dieser Situation herausholt. Wie wir bei unseren Patienten geht der „Captain“ nach folgenden Schritten vor: Bestandsaufnahme - Analyse - Entscheidung und Umsetzung. Schauen wir uns dies übertragen auf unser Arbeitsfeld mal genauer an.

\section{Bestandsaufnahme}

$\rightarrow$ Wie ist der aktuelle Status quo? (Finanzencheck; Rücklagen; Fördermittel)

$\rightarrow$ Welche Therapien sind durchführbar und wie?

$\rightarrow$ Was kann weiterlaufen?

$\rightarrow$ Was geht nicht?

$\rightarrow$ Was geht stattdessen?

$\rightarrow$ In welchem Bereich kann ich Flexibilität aufbauen oder erweitern?

Analyse

$\rightarrow$ Welche „Lecks" hat das „Schiff"?

$\rightarrow$ Sind diese Lecks kurzfristig überbrückbar oder laden wir uns mit dem Aufrechterhalten von bestimmten Kostenpunkten eine ganze Ladung Holzwürmer an Bord, die nicht lange brauchen, um die ganze Seereise zunichte zu machen?

$\rightarrow$ Können wir mit bestimmten Bereichen pausieren? Also können die Mitarbeiter, die in den Heimen therapiert haben, nun recht kurzfristig andere Bereiche erweitern?

\section{Entscheidungen treffen und umsetzen}

So vage unser Weitblick gerade ist: Wir stehen in der Verantwortung für unser Unternehmen. Leider müssen da auch hin und wieder Entscheidungen getroffen werden, zu denen gerade wir empathischen Therapeutenwesen oft keinerlei Muße haben: rein faktische Vernunftentscheidungen, die unangenehm sind und die wir am liebsten vermeiden würden. Unter Umständen kann es sein, dass wir nach der genauen Analyse feststellen, dass ein bestimmter Bereich, und sei er uns auch noch so lieb und teuer, eben nur noch das ist: teuer! Wie lange Sie sich persönlich dies dann erlauben können und wollen, müssen Sie mit den Beratern Ihres Vertrauens besprechen. Um die Entscheidung kommen Sie schlussendlich nicht herum. (Auch wenn vielen Praxen der „Rettungsschirm" trotz all seiner Löcher wirklich geholfen hat, gehe ich persönlich davon aus, dass es keinen zweiten dieser Art geben wird, sollte es zu einem erneuten Krisenfall kommen. Ich freue mich sehr, falls sich dies als falsch erweisen sollte.)

Und wenn Sie eine Entscheidung getroffen haben, müssen Sie sie nur noch in die Tat umsetzen.

\section{$\vec{H}$ Hinweis}

\section{Achtung - nichts ist sicher ...}

Vor Kurzem gab es in Nordrhein-Westfalen die Information, dass wir - unter Beachtung vieler Hygienevorschiften und Abläufe - wieder unsere Patienten in den Pflegeheimen behandeln dürfen. Bei aller Freude und Erleichterung bleibt dies jedoch recht dünnes Eis, das unser Unternehmen nur bedingt tragen kann. Denn nur vier Tage später wurde ein Wohnheim in Köln aufgrund eines Infektionsfalls erneut komplett gesperrt ...

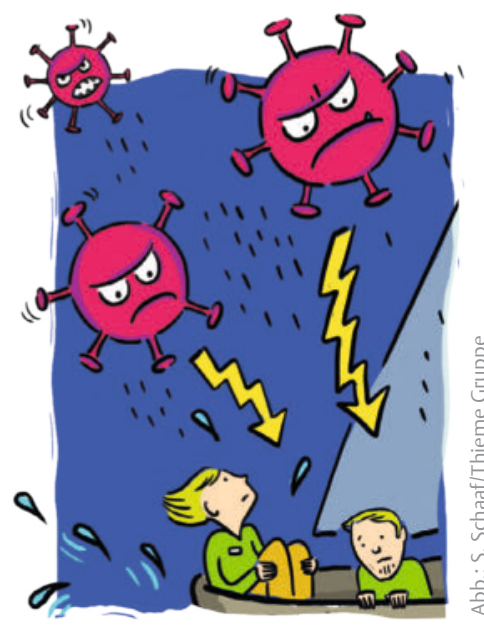




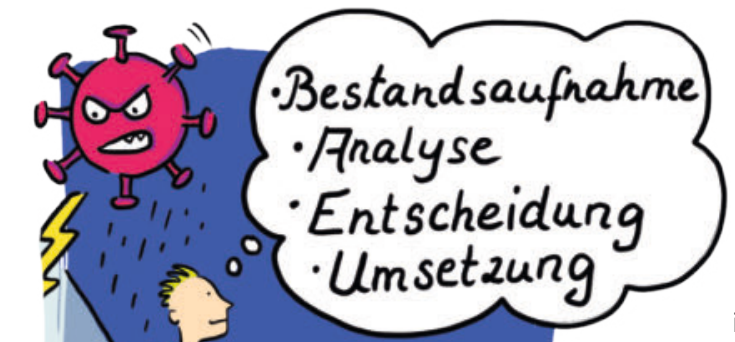

Wie wäre es, wenn Sie mir Ihre „Hühnersuppe für Praxisinhaber“ schicken? Vielleicht gibt es auch einige Mitarbeiter, denen eine „Hühnersuppe für Therapeuten“ einfällt?

Eine erste Geschichte möchte ich gern mit Ihnen teilen: Es war wohl in der dritten Woche des CoronaWahnsinns, als ich von einer Patientin reich beschenkt wurde. Zwar war die Praxis keinen Tag wirklich geschlossen, jedoch hatten wir so massive Ausfälle, dass es in den Räumen leer und still war. Da Sie mich persönlich vermutlich nicht kennen, muss ich dazu sagen, dass leer und vor allem still weder zu mir, noch zu meinem Team passt. Bei uns ist es gewöhnlich laut, lustig, turbulent, man hört Lachen, Telefonklingeln ... und wenn das nicht reicht, singe ich auch gern noch dazu. Aber nun war es still. Es war ein ganz normaler Montag, und ich war allein in den Räumen. Seit mehreren Tagen versuchte ich, eine Patientin telefonisch zu erreichen, um ihren Termin abzusagen, denn sie gehört zumindest aufgrund des Alters zur Risikogruppe. Kein Anrufbeantworter, kein Rückruf. Um zwölf Uhr klingelt es an der Tür und diese ältere Dame steht vor mir. Sie sagte: „Ich weiß, dass Sie mich angerufen haben. Ich weiß auch warum. Sie wollten mir absagen. Das will ich aber nicht. Ich will hier sein! Sie tun mir

\section{פy} mich ständig daran, auf eine Situation, ein Ereignis, eine Krise oder auch den Alltag noch mal von einer anderen Seite zu schauen.

Dazu fallen mir die

\section{Gerade Therapeuten wissen, wie man Krisen übersteht.} Bände „Hühnersuppe für die Seele" von Jack Canfield ein. Er hat irgendwann einmal entdeckt, dass es in jeder Familie Geschichten gibt, die der Seele guttun, sie ernähren und wärmen wie eine Hühnersuppe, wenn man sich eine Erkältung eingefangen hat. Geschichten über wiedergefundene Freunde, Glücksmomente, wunderbare Zufälle. Inzwischen hat Jack Canfield eine Unmenge Nachfolgebücher herausgegeben, die sich nur aus den Geschichten zusammensetzen, die Menschen ihm geschickt haben. Er verteilt diese Geschichten, auf dass sich auch andere daran erwärmen können, Mut schöpfen oder auch schmunzeln können. gut, Ihre Therapie tut mir gut. Und jetzt tue ich Ihnen gut und sage diesen Termin eben gerade nicht ab. Dass es hier so still ist, hab ich mir schon gedacht." Daraufhin nimmt sie ihr Smartphone heraus und lässt „Ode an die Freude“ in donnernder Lautstärke durch die Praxis schallen. Die Patientin grinst ganz breit, weil ich da mit Tränen in den Augen vor ihr stehe, und sagt dann noch: „So, jetzt lassen Sie mal ein paar ordentliche Götterfunken in meinen Handgelenken los, die haben das nötig!“

Leisten Sie Aufbau-Arbeit $\rightarrow$ So viel Zeit mussten wir in den letzten Wochen mit Sorgen, Analysen, Nachrichten etc. verbringen. Da haben wir viele Federn gelassen. Nun sollten wir wieder etwas tun, das uns, unser Selbstvertrauen und unsere Zuversicht wieder aufbaut. Daher mein Aufruf: Schreiben Sie mir an Barbara.Freitag@ gmx.net. Was haben Sie Tolles, Berührendes

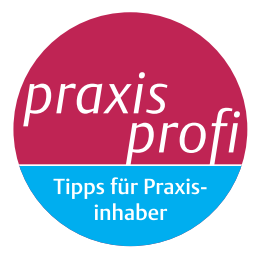
erlebt, welche cleveren

Entscheidungen haben Sie getroffen, was hat sich wunderbar ergeben und entwickelt? Fragen Sie sich: Wofür war diese ganze Sache eigentlich gut? Was habe ich daraus gelernt? Was habe ich gewonnen? Was will ich beibehalten?

Nicht nur ich bin darauf gespannt - Ihre Kollegen sind es auch! Darum möchte ich in

\section{5}

\section{Tun Sie etwas, das Ihr Selbstvertrauen und Ihre Zuversicht wieder aufbaut.}

einem nächsten Artikel von Ihren „Hühnersuppenerlebnissen“, also Seelen- und Herzwärmern, Mutmachern, genialen Ideen und glücklichen Zufällen berichten (natürlich anonymisiere ich Ihre Zuschriften). Damit auch andere an diesen kleinen wunderbaren Episoden teilhaben können.

In einer Krise zu stecken, ist schon schlimm genug, aber damit auch noch gefühlt allein in der Verantwortung zu sein, ist grauenhaft. Stärken wir Therapeuten uns also gegenseitig, auch wenn viele Kilometer und auch Heilmittelbranchen zwischen uns liegen. Durch diesen Sturm mit all seinen Nachwehen kommen wir nur gemeinsam! Barbara Freitag-Herse

\section{Autorin}

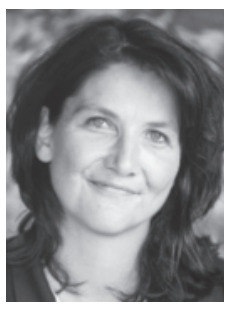

Barbara Freitag-Herse ist selbstständige Ergotherapeutin, Coach, Dozentin und Kommunikationstrainerin. Seit vielen Jahren begleitet sie therapeutische und pädagogische Teams in Findungs- und Konfliktsituationen. Hier und auch in den Familiencoachings liegt ihr besonders der wertschätzende und gleichwürdige Umgang miteinander am Herzen. „Gemeinsam zu Begeisterung, Lachen und Entwicklung “ ist ihr Grundthema bei Workshops und Seminaren. 